\title{
FORMATION OF P CYGNI PROFILES WITH LIMB DARKENING OF THE STELLAR CORE
}

\author{
JEAN SURDEJ* \\ Institut d'Astrophysique, Cointe-Ougrée, Belgium
}

(Received 16 February, 1981)

\begin{abstract}
In the framework of the Sobolev approximation, we investigate the effects of limb darkening of the stellar core onto the formation of line profiles in rapidly expanding envelopes.

For the case of outward-accelerating envelopes, it is shown that the $\mathbf{P}$ Cygni profiles, calculated with and without limb darkening, do appear almost identical as long as the size of the envelope remains large with respect to the radius of the star. As the extent of the envelope decreases, the resulting profiles are found to differ appreciably close to the line center.

When considering outward-decelerating (or equivalently inward-accelerating) envelopes, calculations with the limb darkening effects reveal that the P Cygni profiles are more strongly affected than in the previous case. These effects are specially important in the blue wing and near the center of the resulting line profiles, irrespective of the size of the envelope.
\end{abstract}

\section{Introduction}

In most of the numerical applications dealing with the formation of line profiles in outward-accelerating envelopes, hereafter referred to as AE (Castor, 1970; Lucy, 1971; Kuan and Kuhi, 1975; Oegerle and van Blerkom, 1976; Castor and Lamers, 1979; Rumpl, 1980; etc.), in outward-decelerating envelopes, henceforth referred to as DE (Kuan and Kuhi, 1975; Grachev and Grinin, 1975; Marti and Noerdlinger, 1977; Rybicki and Hummer, 1978; Surdej, 1979; Surdej and Swings, 1981; etc.) and, equivalently, in inward-accelerating envelopes (Bertout, 1977; etc.), the author implicitly assumes that the central stellar core radiates a photospheric continuum spectrum without limb darkening.

On the basis of a few calculated line profiles in AE envelopes, Castor and Lamers (1979) conclude that, except when very close to the line center, the effects of limb darkening are not important when observed profiles are compared with predicted ones. Similarly, Grachev and Grinin (1975) report that P Cygni profiles formed in DE envelopes are not very sensitive to the choice of the limb darkening law.

In order to clearly set out the physical and/or geometrical conditions under which the effects of limb darkening play a major role in the formation of P Cygni profiles, extensive calculations were made using Sobolev-type approximations for the transfer of line photons. In the frame of a two-level atom model, we

* Chargé de Recherches au Fonds National de la Recherche Scientifique, Belgium. 
hereafter present some of these calculations for the cases of $\mathrm{AE}$ and $\mathrm{DE}$ envelopes as well as a discussion of the results.

\section{Basic Model}

We shall first briefly recall the general hypotheses assumed here when dealing with the formation of line profiles in the context of the Sobolev approximation (Sobolev, 1947, 1957, 1958). A more detailed discussion of these may be found in various articles, such as those by Marti and Noerdlinger (1977), Rybicki and Hummer (1978), and Surdej (1977, 1978, 1979; respectively, hereafter referred to as Papers I, II, III).

In the remainder of this paper, we consider either spherical envelopes around a stellar core, which are in rapid expansion or contraction, with either a positive or negative radial velocity gradient. The level-populations of the flowing atoms have reached a steady state and, in the frame of a moving atom, the emitted photons are completely redistributed in frequency and direction. To avoid any further complexities, only radiative exchanges in the single-line transition $1 \leftrightarrows 2$ are taken into account and no attempt is made to cover the ionization balance of the atomic species across the medium.

In the following, we adopt velocity distributions of the type

$$
v(r)=v_{0} L^{-l}
$$

where

$$
L=r / R^{*}
$$

expresses the radial distance $r$ to the central star in stellar radii $R^{*}$ units. $v_{0}$, a positive (or negative) constant, represents the radial velocity at the stellar surface for flows directed outwardly (or inwardly) and $l$ is an accelerating (or decelerating) parameter when negative (or positive). For the case of DE envelopes, we shall also consider the velocity fields

$$
v(r)=v_{\infty} \sqrt{1+G / L}
$$

with the decelerating parameter $G$ given by

$$
G=\left(\frac{v_{0}}{v_{\infty}}\right)^{2}-1
$$

which describe the motion of the fluid ejected (or collapsing) at the stellar surface with a velocity $v_{0}$ and, further on, decelerated (or accelerated) in the gravitational field of the central object. In these equations $v_{\infty}$ represents the 
terminal (or initial) velocity; and we should note that, for the case $v_{\infty}=0$, the velocity field given by Equation (3) is identical to that in (1) for the value of the decelerating parameter $l=0.5$.

When estimating the limb darkening effects onto the formation of $\mathrm{P}$ Cygni profiles in $\mathrm{AE}$ and $\mathrm{DE}$ envelopes, we shall assume that the quadratic law

$$
I_{c}\left(\theta^{*}\right)=I_{c}\left[1-U_{1}-U_{2}+U_{1} \cos \left(\theta^{*}\right)+U_{2} \cos ^{2}\left(\theta^{*}\right)\right]
$$

represents the limb-darkening of the central stellar core (cf. Castor and Lamers, 1979). In that expression, $U_{1}$ and $U_{2}$ stand for the limb darkening factors and $\theta^{*}$ is the angle between the direction of radiation and the normal to the stellar surface. For convenience, we shall denote the limb darkening law by $\psi\left(\theta^{*}\right)$, so that

$$
I_{c}\left(\theta^{*}\right)=I_{c} \psi\left(\theta^{*}\right)
$$

Calculations of P Cygni profiles with limb darkening of the stellar core are easily performed by using an approach similar to the one followed when deriving the expression of line profiles formed in the absence of limb darkening. Two steps can be distinguished by that method. In the first one, we solve the statistical equilibrium equation (cf., Paper I) for a two-level atom model in order to evaluate the source function $S_{12}$ and the fictive optical depth $\tau_{12}$ across the atmosphere (cf., Paper II). The physical conditions having been determined over the whole space of the medium, it is then possible to derive and calculate the expression of the line profile $E(X) / E_{c}$ formed in rapidly expanding (resp. contracting) envelopes (cf., Paper III). On the basis of these derived expressions, it is straightforward to establish the relevant equations for the source function $S_{12}$ and the line profile function $E(X) / E_{c}$ when allowance is made for the limb-darkening effects. Adopting the formalism used in previous works (see Papers I, II, III), we establish the form of these equations in the next section.

\section{Equations for the Line Profile and Source Functions}

Because the transfer of line radiation in AE envelopes is purely local (cf. Castor, 1970; Lucy, 1971), whereas radiative interactions between distant parts of the atmosphere also take place in DE envelopes (cf. Grachev and Grinin, 1975; Marti and Noerdlinger, 1977) we shall consider separately both cases of AE and DE envelopes when deriving the equations for the line profile and source functions in presence of limb darkening effects.

\subsection{AE ENVELOPES}

With the dimensionless frequency $X$ defined by

$$
X=-\frac{v(r)}{v_{\max }} \cos (\theta)
$$


where $v(r) \cos (\theta)$ represents the macroscopic velocity of the medium, at a distance $L$, projected along the line of sight making an angle $\theta$ with the radial direction (see Figure 1) and where $v_{\max }$ stands for the maximal expansion velocity of the atmosphere, one easily finds that the line profile function

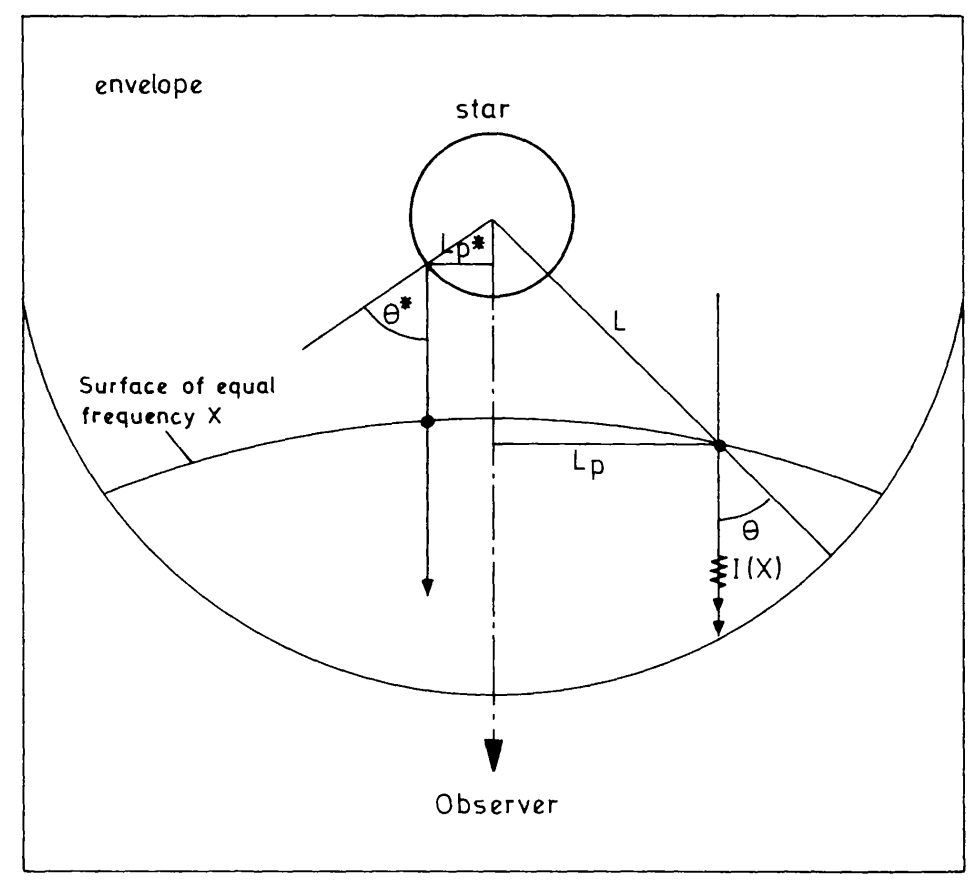

Fig. 1. Intersections between a surface of equal frequency $X$ and the axes $\left(L_{p}, L_{p}^{*}\right)$ parallel to the line of sight along which is studied the transfer of line radiation.

$E(X) / E_{c}$ expresses the new energy distribution of the stellar continuum in the frequency interval $X \in[-1,1] . E(X) / E_{c}$ merely represents the total amount of spectral energy $E(X)$ defined per frequency, and solid angle units radiated by the medium towards a fixed observer, and which has been normalized to the flux $E_{c}$ of the stellar continuum $I_{c}\left(\theta^{*}\right)$ integrated over the surface of the stellar disk, i.e.,

$$
E_{c}=\Pi \overline{I_{c}}
$$

with

$$
\overline{I_{c}}=2 I_{c} \int_{0}^{1} \psi\left(L_{p}^{*}\right) L_{p}^{*} \mathrm{~d} L_{p}^{*}
$$

and

$$
L_{p}^{*}=\sin \left(\theta^{*}\right)
$$

assuming that $R^{*}=1$. From relation (9), one deduces that $\overline{I_{c}}=I_{c}$, in the absence of limb darkening. 
The quantity $E(X)$ is itself given by the integration of the monochromatic function $I(X)$, implicitly space defined, over a plane perpendicular to the line of sight. Due to the symmetry of the envelope around the line of sight, we have

$$
E(X)=2 \Pi \int_{0}^{L_{\max }} I(X) L_{p} \mathrm{~d} L_{p},
$$

with

$$
L_{p}=L \sin (\theta),
$$

and where $I(X)$ is evaluated over those parts of the atmosphere presenting a constant Doppler shift $v(r) \cos (\theta)$ with respect to the observer (see relation (7)). This geometrical locus also defines the so-called surfaces of equal frequency $X$.

For given values of the impact parameter $L p \in\left[0, L_{\max }\right]$ and of the frequency $X \in[-1,1]$, one easily finds (cf., relation (III. 30)) that the contribution by the scattering atoms to the monochromatic intensity function is

$$
I(X)=S_{12}\left(1-\exp \left(-\tau_{12}\right)\right),
$$

and that for $L_{p}^{*} \in[0,1]$ and $X \in[-1,0[$, the stellar core contribution (cf., relation (III.31)) becomes

$$
I(X)=I_{c} \psi\left(L_{p}^{*}\right) \exp \left(-\tau_{12}\right) .
$$

In these last two relations, the quantities $S_{12}$ and $\tau_{12}$ are evaluated at the intersection between the surface of equal frequency $X$ and the axis parallel to the line of sight for the given value of the impact parameter $L_{p}\left(\right.$ or $\left.L_{p}^{*}\right)$.

Let us point out that in relation (13), the source function $S_{12}$ is still given (cf., relation (II.18)) by

$$
S_{12}=I_{c} \beta_{12}^{3} / \beta_{12}^{1},
$$

where

$$
\beta_{12}^{1}=\int_{\Omega=4 \Pi} \frac{\left(1-\exp \left(-\tau_{12}\right)\right)}{\tau_{12}} \frac{d \omega}{4 \Pi}
$$

represents the probability for a photon created in the line transition $2 \rightarrow 1$ to escape locally the medium along any direction. However, the quantity $\beta_{12}^{3}$ evaluated at a given point $R\left(L_{p}, X\right)$ in the envelope (cf., relation (II.16)), is now expressed by

$$
\beta_{12}^{3}=\int_{\Omega=4 \Pi W} \psi\left(\theta^{*}\right) \frac{\left(1-\exp \left(-\tau_{12}\right)\right)}{\tau_{12}} \frac{\mathrm{d} \omega}{4 \Pi},
$$


and represents the probability that a stellar photon emitted along any direction $\theta^{*} \in[0, \Pi / 2]$ with a limb darkening $\psi\left(\theta^{*}\right)$ will interact with atoms at $R$. We recall that, in this equation, $W$ stands for the geometrical dilution factor calculated at the point $R$. In order to perform the last integration, it appears very useful to set the relations existing between the angles $\theta, \theta^{*}$ and the solid angle $\omega$ :

$$
\omega=2 \Pi(1-\cos (\theta)) \text {, }
$$

and

$$
\sin \left(\theta^{*}\right)=L \sin (\theta) .
$$

If we combine Equations (8)-(14), the expression for the line profile function in $\mathrm{AE}$ envelopes reduces to

$$
\begin{aligned}
& \frac{E(X)}{E_{c}}= 2\left[\int_{0}^{L_{\max }} \frac{S_{12}}{\overline{I_{c}}}\left(1-\exp \left(-\tau_{12}\right)\right) L_{p} \mathrm{~d} L_{p}+\right. \\
&\left.+\frac{I_{c}}{\bar{I}_{c}} \int_{0}^{1} \psi\left(L_{p}^{*}\right) \exp \left(-\tau_{12}\right) L_{p}^{*} \mathrm{~d} L_{p}^{*}\right], \quad \text { if } \quad X \in[-1,0[, \\
& \frac{E(X)}{E_{c}}=2\left[\int_{1}^{L_{\max }} \frac{S_{12}}{\overline{I_{c}}}\left(1-\exp \left(-\tau_{12}\right)\right) L_{p} \mathrm{~d} L_{p}+\frac{1}{2}\right], \quad \text { if } X \in[0,1] .
\end{aligned}
$$

\subsection{DE ENVELOPES}

Whereas, in the case of $\mathrm{AE}$ envelopes there is, at maximum, one intersection between a surface of equal frequency $X$ and a direction parallel to the line of sight, two such intersections may occur in DE envelopes (see Figures 6-11 in Paper III). Keeping in mind these possible interactions between distant parts of the atmosphere and denoting by $R(L, \theta)$ and $R^{\prime}\left(L^{\prime}>L, \theta^{\prime}\right)$ two such distant interacting points, we can easily modify Equation (20) in order to express the line profile function in DE envelopes. We obtain

$$
\begin{aligned}
\frac{E(X)}{E_{c}}= & 2\left[\int _ { 0 } ^ { L _ { \operatorname { m a x } } } \left(\frac{S_{12}}{\overline{I_{c}}}\left(1-\exp \left(-\tau_{12}\right)\right) \exp \left(-\tau_{12}^{\prime}\right)+\right.\right. \\
& \left.+\frac{S_{12}^{\prime}}{\overline{I_{c}}}\left(1-\exp \left(-\tau_{12}^{\prime}\right)\right)\right) L_{p} \mathrm{~d} L_{p}+ \\
& \left.+\frac{I_{c}}{\overline{I_{c}}} \int_{0}^{1} \psi\left(L_{p}^{*}\right) \exp \left(-\tau_{12}\right) \exp \left(-\tau_{12}^{\prime}\right) L_{p}^{*} \mathrm{~d} L_{p}^{*}\right], \quad \text { if } X \in[-1,0[, \\
\frac{E(X)}{E_{c}}= & 2\left[\int _ { 1 } ^ { L _ { \operatorname { m a x } } } \left(\frac{S_{12}^{\prime}}{\overline{I_{c}}}\left(1-\exp \left(-\tau_{12}^{\prime}\right)\right) \exp \left(-\tau_{12}\right)+\right.\right. \\
& \left.\left.+\frac{S_{12}}{\overline{I_{c}}}\left(1-\exp \left(-\tau_{12}\right)\right)\right) L_{p} \mathrm{~d} L_{p}+\frac{1}{2}\right], \quad \text { if } \quad X \in[0,1] .
\end{aligned}
$$


If, for given values of the impact parameter $L_{p} \in\left[0, L_{\max }\right]$ and of the frequency $X \in[-1,1]$, Equation (7) admits only one (resp. zero) solution, the fictive opacities $\tau_{12}^{\prime}$ (resp. $\tau_{12}$ and $\tau_{12}^{\prime}$ ) in Equation (21) are set equal to zero.

The expression for the source function $S_{12}$ (or $S_{12}^{\prime}$ ) appearing in Equation (21) is easily found (cf., relation (II.29)) to be

$$
S_{12}=\frac{\beta_{12}^{5}}{\beta_{12}^{1}} I_{c}+\frac{1}{\beta_{12}^{1}} \int_{\Omega\left(R, R^{\prime}\right)} S_{12}^{\prime}\left(1-\exp \left(-\tau_{12}^{\prime}\right)\right) \frac{\left(1-\exp \left(-\tau_{12}\right)\right)}{\tau_{12}} \frac{\mathrm{d} \omega}{4 \Pi},
$$

with

$$
\beta_{12}^{5}=\int_{\Omega=4 \Pi W} \psi\left(\theta^{*}\right) \frac{\left(1-\exp \left(-\tau_{12}\right)\right)}{\tau_{12}} \exp \left(-\tau_{12}^{\prime}\right) \frac{\mathrm{d} \omega}{4 \Pi}
$$

We recall that in these relations, the quantities $S_{12}^{\prime}, \tau_{12}^{\prime}$ are calculated at distant points $R^{\prime}$ whose relative velocity to $R$ is null. The integration over the solid angle $\Omega\left(R, R^{\prime}\right)$ in Equation (22) encompasses all directions from $R$ such that a couple of points $\left(R, R^{\prime}\right)$ exist. We refer the reader to Paper II for more details about the numerical solution of Equation (22).

\section{Numerical Applications}

For various values of the limb darkening factors $U_{1}$ and $U_{2}$, we have performed extensive calculations of line profiles in $\mathrm{AE}$ and $\mathrm{DE}$ envelopes.

Adopting the opacity distributions (cf., relation (II.42))

$$
\tau_{12}=\tau_{12}^{l} L^{2 l-1} F(\theta)\left(1-\left(\frac{g_{1} n_{2}}{g_{2} n_{1}}\right)\right) /\left(1+\frac{n_{2}}{n_{1}}\right)
$$

with

$$
F(\theta)=\left|\frac{l}{1-(l+1) \cos ^{2}(\theta)}\right|
$$

for the velocity fields given in (1); and, similarly,

$$
\tau_{12}=\tau_{12}^{l} F(L, \theta)\left(1-\left(\frac{g_{1} n_{2}}{g_{2} n_{1}}\right)\right) /\left(1+\frac{n_{2}}{n_{1}}\right)
$$

with

$$
F(L, \theta)=\frac{1}{\left|2(L / G+1)-(2 L / G+3) \cos ^{2}(\theta)\right|}
$$

for the velocity distributions given in (3), we have first determined the physical conditions $\left(S_{12}, \tau_{12}\right)$ prevailing throughout the expanding envelopes (see Equations (15) or (22)). We recall that $n_{1}, n_{2}$ and $g_{1}, g_{2}$ denote, respectively, the volume populations and the statistical weights of the atomic levels 1 and 2 , and 
that the parameter $\tau_{12}^{l}$ is the radial $(\theta=0)$ optical depth at the stellar surface $(L=1)$.

From these results and by means of Equations (21) or (22), it is then straightforward to calculate the line profile function $E(X) / E_{c}$ in the frequency interval $X \in[-1,1]$.

For both cases of $\mathrm{AE}$ and $\mathrm{DE}$ envelopes, we have illustrated in Figures 2-7 some of these calculated line profiles which, as a whole, include the various effects arising from limb-darkening of the stellar core. The physical and

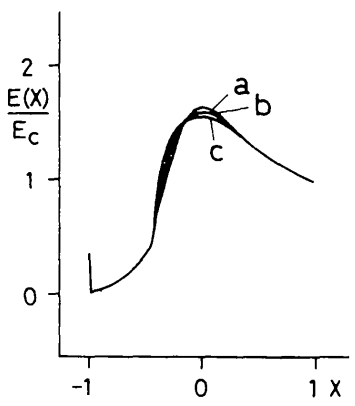

Fig. 2.

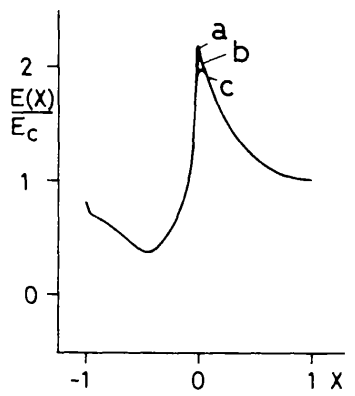

Fig. 4.

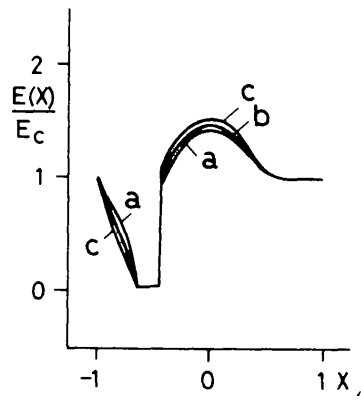

Fig. 6.

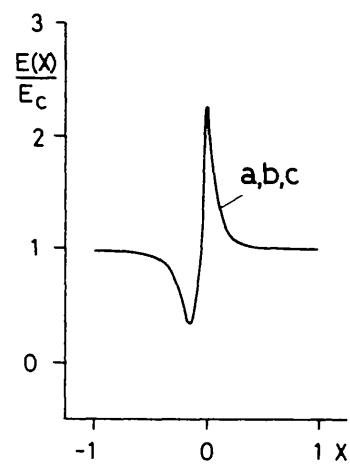

$445 \quad$ Fig. 3.

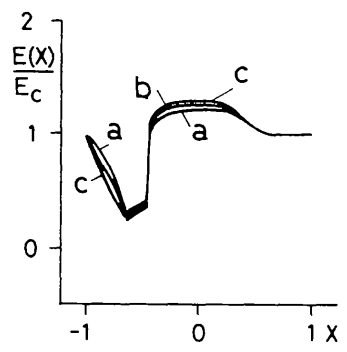

Fig. 5.

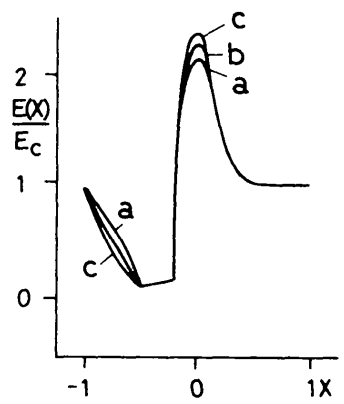

Fig. 7. 
geometrical parameters characterizing each of the line profiles are summarized in Table I. Also, the labels a, b, c indicated in each of these figures refer to the distinct limb-darkening laws as follows:

$$
\begin{aligned}
& \text { a }: \psi\left(\theta^{*}\right)=1\left(U_{1}=0, U_{2}=0\right) \text {, i.e., no limb-darkening; } \\
& \text { b }: \psi\left(\theta^{*}\right)=\cos \left(\theta^{*}\right)\left(U_{1}=1, U_{2}=0\right) \text {, i.e., a mild limb-darkening; } \\
& \text { c }: \psi\left(\theta^{*}\right)=\cos ^{2}\left(\theta^{*}\right)\left(U_{1}=0, U_{2}=1\right) \text {, i.e., a strong limb-darkening. }
\end{aligned}
$$

TABLE I

Caption for Figures 2-7

\begin{tabular}{cccr}
\hline Figure & $l$ (or $G)$ & $\tau_{12}^{l}$ & $L_{\max }$ \\
\hline 2 & -0.5 & $10^{9}$ & 5 \\
3 & -1 & $10^{3}$ & 50 \\
4 & -2 & $10^{3}$ & 5 \\
5 & $G=400$ & 1 & 5 \\
6 & 0.5 & 10 & 5 \\
7 & 1 & 10 & 5 \\
\hline
\end{tabular}

\section{Discussion}

\subsection{AE ENVELOPES}

Comparing, the profiles labelled a, b, c in Figures 2-4, we conclude that the effects of limb-darkening of the stellar core in AE envelopes are not very significant, unless the size of the envelope is of the order of only a few stellar radii. This conclusion can be directly explained as follows: (i) The quantity $S_{12} / \overline{I_{c}}$ which enters Equation (20) of the line profile function, is almost independent of the limb darkening of the stellar core. Indeed, for a given value of the impact parameter $L_{p}^{*}=\sin \left(\theta_{0}^{*}\right)$, the fraction of the stellar disk, as seen by a fixed observer, which emits radiation along directions such as $\theta^{*}<\theta^{*}$ is equal to $\sin ^{2}\left(\theta_{0}^{*}\right)$. It is now straightforward to establish that the quantity $S_{12} / \overline{I_{c}}$, evaluated at a distance $L$, will be free of limb-darkening effects if the ratio $F$ of the solid angle subtending rays emerging from the stellar core along directions $\theta^{*} \leqslant \theta_{0}^{*}$ to the solid angle $\Omega=4 \Pi W$, is also equal to $\sin ^{2}\left(\theta^{*}\right)$. This ratio may be written as (see Equations (18) and (19))

$$
F=\frac{1}{2}\left(1-\sqrt{1-\frac{\sin ^{2}\left(\theta_{0}^{*}\right)}{L^{2}}}\right) / W
$$

where the geometrical dilution factor is expressed by

$$
W=\frac{1}{2}\left(1-\sqrt{1-\frac{1}{L^{2}}}\right) .
$$


Rigorously, we have

$$
F<\sin ^{2}\left(\theta_{0}^{*}\right)
$$

but to the second-order approximation we find that, for $L \gg 1$,

$$
W=\frac{1}{4} L^{2},
$$

and similarly that

$$
F=\sin ^{2}\left(\theta^{*}\right) \text {. }
$$

The physical meaning of these results is that the effects of limb darkening are only important in the close vicinity of the star and that the quantity $S_{12} / \overline{I_{c}}$ appears there slightly decreased when compared to its value calculated without limb darkening.

We shall now give a more quantitative evaluation of the departure between the quantities $S_{12} / \overline{I_{c}}$ and $S_{12} / I_{c}$ calculated with and without limb darkening effects, respectively. Considering the particular case $l=-1$ (isotropy!) and with the help of relations (15)-(17), we may write

$$
S_{12}=I_{c} \int_{\Omega=4 \Pi W} \psi\left(\theta^{*}\right) \frac{\mathrm{d} \omega}{4 \Pi} .
$$

For the strong limb darkening law, i.e., $\psi\left(\theta^{*}\right)=\cos ^{2}\left(\theta^{*}\right)$, and with the use of relations (18) and (19), the last expression reduces to

$$
S_{12}=I_{c}\left(\frac{1}{2}\left(1-L^{2}\right)\left(1-\sqrt{1-\left(\frac{1}{L}\right)^{2}}\right)+\frac{L^{2}}{6}\left(1-\left(1-\left(\frac{1}{L}\right)^{2}\right)^{3 / 2}\right)\right) .
$$

To second-order approximation for $L \gg 1$, we find that

$$
S_{12} \simeq I_{c} W / 2,
$$

where the geometrical dilution factor is approximated by (31). With the definition (9) for $\overline{I_{C}}$, we obtain

$$
\overline{I_{c}}=I_{c} / 2,
$$

and finally

$$
\frac{S_{12}}{\overline{I_{c}}} \simeq W
$$

This result can be directly compared with the rigorous one $S_{12} / I_{c}=W$, obtained for the case $\psi\left(\theta^{*}\right)=1$. In reality, the relative error between the exact value of $S_{12} / \overline{I_{c}}$ (see relation (34)) and that of $S_{12} / I_{c}$ amounts to $33 \%$ at $L=1,5 \%$ at $L=1.5$ and is already much smaller than $1 \%$ for $L \geqslant 3$.

(ii) Since the quantities $S_{12} / \overline{I_{c}}\left(\psi\left(\theta^{*}\right) \neq 1\right)$ and $S_{12} / I_{c}\left(\psi\left(\theta^{*}\right)=1\right)$ only differ appreciably within a small region nearby the central star, we understand why in Figures $2-4$ the resulting profiles appear distinct only in the vicinity of the line 
center (cf., Castor and Lamers, 1979). Indeed, the surfaces of equal frequency $X \approx 0$ are the only ones being partially located in the neighbourhood of the stellar core (cf., Figures 6-8 in Paper III).

Furthermore, because the spatial unfolding of the surfaces of equal frequency $X$ depends on the value of the accelerating parameter $l$, it is easy to state that the frequency interval of the line profile affected by the limb darkening of the star will be greater for those velocity fields which are characterized by the presence of a larger radial velocity gradient at the basis of the envelope (cf., Figure 2). Also, in that case, the partial occultation of the stellar disk by the surfaces of equal frequency $X \in\left[-v_{0} / v_{\max }, 0\right]$ will be the most apparent and this explains why in Figure 2 the blue wing of the line profile appears somewhat enhanced as the limb darkening is steeper.

Finally, because the extent of the surfaces of equal frequency $X \approx 0$ increases almost linearly with the value $L_{\max }$, one expects the effects of limb darkening to become negligible for large values of $L_{\max }$ (see Figure $3, L_{\max }=50$ ).

\subsection{DE ENVELOPES}

Inspection of Figures 5-7 shows that the line profiles formed in DE envelopes are more strongly affected by the limb darkening of the stellar core.

As the limb darkening increases, so the line profile function $E(X) / E_{c}$ appears to be enhanced close to the line center. This departure is essentially due to the fact that the value of the quantity $S_{12} / \overline{I_{c}}$ (see Equation (21)) increases everywhere in the envelope with the importance of the limb darkening. Considering, for instance, the line profiles labelled a and $\mathrm{c}$ in Figure $7\left(l=1, \tau_{12} \gg 1\right)$, we can readily show that it so. Indeed, recalling the expression (22) for the source function $S_{12}$, it is just necessary to demonstrate that

$$
\begin{aligned}
\int_{\Omega=4 \Pi W} \frac{\left(1-\exp \left(-\tau_{12}\right)\right)}{\tau_{12}} & \exp \left(-\tau_{12}^{\prime}\right) 2 \cos ^{2}\left(\theta^{*}\right) \frac{\mathrm{d} \omega}{4 \Pi}> \\
& >\int_{\Omega=4 \Pi W} \frac{\left(1-\exp \left(-\tau_{12}\right)\right)}{\tau_{12}} \exp \left(-\tau_{12}^{\prime}\right) \frac{\mathrm{d} \omega}{4 \Pi}
\end{aligned}
$$

or when making use of relations (18) and (19), that

$$
\begin{aligned}
\int_{0}^{\theta_{\text {inf }}} \frac{\left(1-\exp \left(-\tau_{12}\right)\right)}{\tau_{12}} 2\left(1-L^{2} \sin ^{2}(\theta)\right) \sin (\theta) \mathrm{d} \theta> & \\
& >\int_{0}^{\theta_{\text {inf }}} \frac{\left(1-\exp \left(-\tau_{12}\right)\right)}{\tau_{12}} \sin (\theta) \mathrm{d} \theta,
\end{aligned}
$$

where (see relation (II.62))

$$
\theta_{\text {inf }}=\arctan (1 / L) \text {. }
$$

The passage from condition (38) to (39) is immediate if we recall that along directions $\theta \in\left[\theta_{\text {inf }}\right.$, arcsin $\left.(1 / L)\right]$ (see Figure 5 in Paper I) the geometrical locus 
of the distant points $R^{\prime}$, whose relative velocity to $R$ is null, occults totally $\left(\tau_{12}^{\prime} \gg 1\right)$ the stellar disk. For condition (39) to be fulfilled, it is sufficient that

$$
\sin (\theta)<\frac{1}{\sqrt{2}} \frac{1}{L}
$$

and according to relation (40), this is always the case as long as $L>1$.

From Figures 5-7, it is also remarkable to note how, in the frequency interval $X \in\left[-1,-X_{c}\right]$, the shape of the P Cygni absorption component varies with the limb darkening law $\psi\left(\theta^{*}\right)$. We recall (cf., relation (III.35)) that $X_{c}$ refers to the value of the frequency over which the surfaces of equal frequency $X$ are totally occulted by the stellar core (see Figures 9-11 in Paper III).

As can be predicted, the shape of the blue wing of the absorption component directly reflects the contribution $E_{\text {abs }}(X) / E_{c}$ of the unscattered stellar radiation to the line profile $E(X) / E_{c}$. Considering the line profiles a, b, c illustrated in Figure $6\left(l=0.5, \tau_{12} \gg 1\right)$ and in Figure $7\left(l=1, \tau_{12} \gg 1\right)$, we show below how this dependence holds.

For $X \in\left[-1,-X_{c}\right]$ we have (see Equation (21))

$$
\frac{E_{\mathrm{abs}}(X)}{E_{c}}=\frac{2 I_{c}}{\overline{I_{c}}} \int_{0}^{1} \psi\left(L_{p}^{*}\right) \exp \left(-\tau_{12}\right) \exp \left(-\tau_{12}^{\prime}\right) L_{p}^{*} \mathrm{~d} L_{p}^{*},
$$

and since the surfaces of equal frequency $X \in\left[-1,-X_{c}\right]$ occult totally $\left(\tau_{12} \gg 1\right.$, $\left.\tau_{12}^{\prime} \gg 1\right)$ the stellar disk between $L_{p}^{*}=0$ and $L_{p}^{*}=L_{p}^{*}(X)$, the previous expression reduces to

$$
\frac{E_{\mathrm{abs}}(X)}{E_{c}}=\frac{2 I_{c}}{\overline{I_{c}}} \int_{L_{p}^{*}(X)}^{1} \psi\left(L_{p}^{*}\right) L_{p}^{*} \mathrm{~d} L_{p}^{*} .
$$

Combining relations (1), (7) and (12), we can express the dependence of the impact parameter $L_{p}^{*}$ as a function of $L$ along a surface of equal frequency $X$

$$
L_{p}^{*}=L \sqrt{1-\left(X L^{l}\right)^{2}} .
$$

Noting that $L_{p}^{*}$ vanishes at the points $L=0$ and $L=X^{-1 / l}$, we can easily state that $L_{p}^{*}$ becomes an extremum at

$$
L^{*}=\left((l+1) X^{2}\right)^{-1 / 2 l} \text {. }
$$

Consequently, if $L^{*} \geqslant 1$, i.e., for $X \in\left[-1 / \sqrt{l+1},-X_{c}\right]$, we find by means of relation (44), that

$$
L_{p}^{*}(X)=\sqrt{\frac{l}{l+1}}\left((l+1) X^{2}\right)^{-1 / 2 l}
$$

and if $L^{*}<1$, i.e., for $X \in[-1,-1 / \sqrt{l+1}[$, that

$$
L_{p}^{*}(X)=\sqrt{1-X^{2}}
$$

For the different limb-darkening laws adopted in Section 4, expression (43) 
transforms, respectively, into

$$
\begin{aligned}
& \text { a: } \frac{E_{\mathrm{abs}}(X)}{E_{c}}=\left(1-L_{p}^{* 2}(X)\right), \\
& \mathrm{b}: \frac{E_{\mathrm{abs}}(X)}{E_{c}}=\left(1-L_{p}^{* 2}(X)\right)^{3 / 2},
\end{aligned}
$$

and

$$
\mathrm{c}: \frac{E_{\mathrm{abs}}(X)}{E_{c}}=\left(1-L_{p}^{* 2}(X)\right)^{2}
$$

Taking into account the results derived in (46) and (47), we have illustrated in Figure $8(l=0.5)$ and in Figure $9(l=1)$ the behaviour of the function $E_{\text {abs }}(X) / E_{c}$ (see relations (48)-(50)) in the spectral interval $X \in\left[-1,-X_{c}\right]$. The results of these numerical applications are seen to be in very good agreement with the calculations of line profiles (i.e., for $X \in\left[-1,-X_{c}\right]$ ) illustrated in Figures 6 and 7.

If we may secure that an observed line profile is effectively formed in a DE envelope (cf., Grachev and Grinin, 1975; Surdej and Swings, 1981), we conclude that the analysis of such a profile enables one to deduce not only the value of the decelerating parameter $l$ (or $G$ ) and the maximum extent $L_{\max }$ of the medium (see Paper III) but still gives us a direct opportunity to estimate the limb darkening of the stellar core on the basis of the exact shape of the observed P Cygni absorption component.

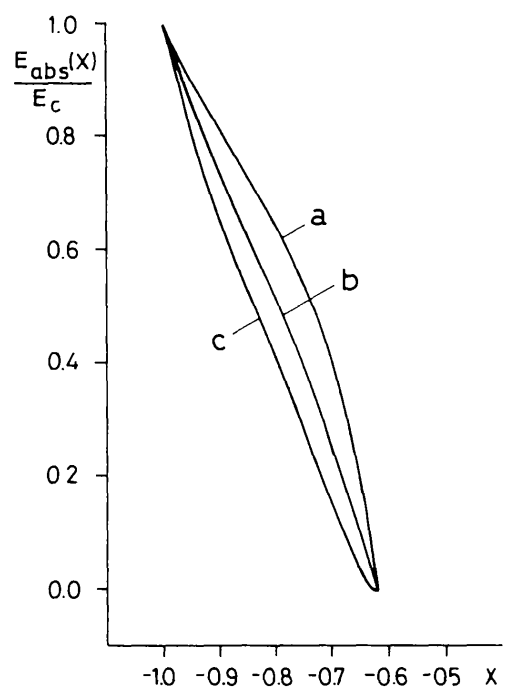

Fig. 8. Behaviour of the absorption profile $E_{\mathrm{abs}}(X) / E_{c}$ in the frequency interval $X \in\left[-1,-X_{c}\right]$ for the limb darkening laws $\psi\left(\theta^{*}\right)=1(\mathrm{a}), \psi\left(\theta^{*}\right)=\cos \left(\theta^{*}\right)(\mathrm{b})$, and $\psi\left(\theta^{*}\right)=\cos ^{2}\left(\theta^{*}\right)(\mathrm{c})$ and for the values of the parameters $l=0.5, \tau_{12} \gg 1$. 


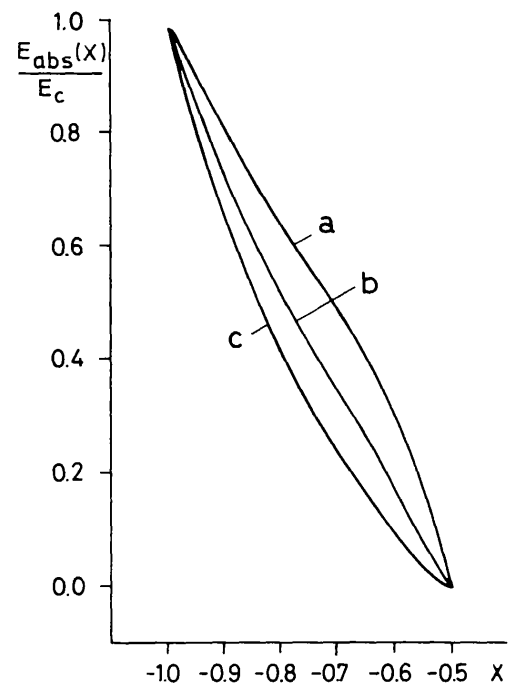

Fig. 9. Behaviour of the absorption profile $E_{\mathrm{abs}}(X) / E_{\mathrm{c}}$ in the frequency interval $X \in\left[-1,-X_{\mathrm{c}}\right]$ for the limb darkening laws $\psi\left(\theta^{*}\right)=1(\mathrm{a}), \psi\left(\theta^{*}\right)=\cos \left(\theta^{*}\right)(\mathrm{b})$, and $\psi\left(\theta^{*}\right)=\cos ^{2}\left(\theta^{*}\right)$ (c) and for the values of the parameters $l=1, \tau_{12} \gg 1$.

\section{Conclusions}

In the frame of a two-level atom model and using Sobolev-type approximations for the transfer of line photons, we have investigated the effects of limb darkening of the stellar core onto the formation of $\mathrm{P}$ Cygni profiles in rapidly expanding envelopes.

For the case of $\mathrm{AE}$ envelopes, we have concluded that the limb darkening effects are unimportant as long as the size $L_{\max }$ of the medium remains large with respect to the radius of the star. However, we have shown that, if the extent of the envelope is of the order of only a few stellar radii, the central part of the emission component can be appreciably weakened and the blue wing of the $P$ Cygni absorption enhanced when compared to calculations without limb darkening. Therefore, when evaluating mass-loss rates from observed P Cygni profiles, much care should be taken in the interpretation of the results on account of these possible effects of the limb darkening.

In $\mathrm{DE}$ envelopes, the radiative coupling between distant parts of the atmosphere acts in such a way that the physical quantity $S_{12} / \overline{I_{c}}$, which determines the intensity of the emission component of the line profile, increases everywhere in the medium with the importance of the limb darkening. Also, the amount of unscattered stellar radiation reaching a fixed observer is shown to be very much dependent on the choice of the limb darkening law. These effects result in affecting appreciably both the emission component and the extreme blue wing of the line profile, irrespective of the maximum extent of the envelope. Finally, another important conclusion that has been drawn is that a thorough analysis of an observed $\mathrm{P}$ Cygni profile formed in a DE envelope allows one to infer the type of darkening law which affects the central source of radiation. 


\section{References}

Bertout, C.: 1977, Astron. Astrophys. 58, 153.

Castor, J. I.: 1970, Monthly Notices Roy. Astron. Soc. 149, 111.

Castor, J. I. and Lamers, H. J. G. L. M.: 1979, Astrophys. J. Suppl. 39, 481.

Grachev, S. I. and Grinin, V. P.: 1975, Astrophysics 11, 20.

Kuan, P. and Kuhi, L. V.: 1975, Astrophys. J. 199, 148.

Lucy, L. B.: 1971, Astrophys. J. 163, 95.

Marti, F. and Noerdlinger, P. D.: 1977, Astrophys. J. 215, 247.

Oegerle, W. R. and van Blerkom, D.: 1976, Astrophys. J. 208, 453.

Rumpl, W. M.: 1980, Astrophys. J. 241, 1055.

Rybicki, G. B. and Hummer, D. G.: 1978, Astrophys. J. 219, 654.

Sobolev, V.V.: 1947, Moving Envelopes of Stars, (in Russian); English translation by S. Gaposchkin, Harvard University Press, Cambridge, Mass., 1960.

Sobolev, V. V.: 1957, Soviet Astron. 1, 678.

Sobolev, V. V.: 1958, in V. A. Ambartsumyan (ed.), Theoretical Astrophysics, Pergamon, London.

Surdej, J.: 1977, Astron. Astrophys. 60, 303 (Paper I).

Surdej, J.: 1978, Astron. Astrophys. 66, 45 (Paper II).

Surdej, J.: 1979, Astron. Astrophys. 73, 1 (Paper III).

Surdej, J. and Swings, J. P.: 1981, Astron. Astrophys., in press. 